\title{
Reduction Formulae for Euclidean Lattice Theories
}

\author{
João C. A. Barata ${ }^{\star}$ \\ Instituto de Física da Universidade de São Paulo, Caixa Postal 20516, Cep. 01498, \\ São Paulo, SP, Brasil
}

Received March 21, 1991

Abstract. LSZ reduction formulae for Euclidean Lattice Theories are presented.

\section{Introduction}

There is an increasing interest in the physics literature in the study of scattering processes in Euclidean Lattice Field Theories (see f.i. [4, 5]). The existence of multiparticle states and of a well defined $S$-matrix for these theories has been established in [1] (see also [10]). An important question which rises in this context is the question of the existence of LSZ reduction formulae for the computation of $S$-matrix elements. This work is devoted to the rigorous derivation of these formulae. For relativistic quantum field theories they were first obtained by Lehmann, Symanzik, and Zimmermann in [3] under special assumptions concerning weak asymptotic limits of the fields. The first rigorous proof in the context of Wightman Quantum Field Theories was given by Hepp in [2]. Here we will follow essentially Hepp's ideas but some adaptations to our case are necessary due to the following two facts. First, [2] makes use of locality (Einstein causality) in a strong sense, a property which is generally not available for Lattice Theories. Second, the clustering of the Wightman functions obtained in [1] (see Theorem 2 below), which is an essential property for the construction of the scattering theory, is not uniform in the whole region of space-like separated points.

In Sect. 2 the notation used and the results of [1] which will be needed are introduced. Section 3 presents a smoothness theorem on suitably smeared expectations of time-ordered products of fields which leads directly to the reduction formulae in Sect. 4. The problems mentioned in the last paragraph manifest in the proof of this smoothness theorem, given in Subsects. 3.1 and 3.2. In this last subsection we show how to compensate the lack of locality with the use of the clustering property.

* Partially supported by CNPq 


\section{Definitions and Previous Results}

The results of this work are valid in the framework of Euclidean Lattice Theories described in [1]. We consider a classical statistical mechanical spin system on a $d+1$-dimensional Euclidean cubic lattice $\mathbb{Z}^{d+1}(d \geqq 1)$ provided with a translation invariant and reflection positive equilibrium state, which we call the vacuum state. As in [1] we denote by $\mathscr{H}$ the Hilbert space of finite energy states and by $\mathscr{H}^{(1)}$ the closed one-particle subspace of $\mathscr{H}$ (whose existence is assumed), on which the relation $\left(e^{-H}-e^{-\omega(P)}\right) \mid \mathscr{H}^{(1)}=0$ holds. Here $\underline{P}$ is the momentum operator, i.e., the infinitesimal generator of space translations, $e^{i P \cdot x}=U(\underline{x}), \operatorname{sp}(\underline{P}) \subset(-\pi, \pi]^{d}$, where

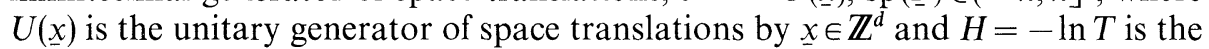
Hamilton operator, $T$ being the infinite volume transfer matrix. Above $\omega$ is the dispersion relation of the particle, which will be assumed to be smooth with $\omega(\underline{p}) \geqq m, \underline{p} \in(-\pi, \pi]^{d}, m$ being the mass gap. We assume $m>0$. We will also denote by $\mathscr{A}$ the $*$-algebra generated by the quasi-local observables with finite energy transfer (see [1]). As discussed in [1] a dense subspace $\mathscr{D}^{(1)}$ of $\mathscr{H}^{(1)}$ is created from the vacuum by almost local operators $A \in \mathscr{A}$ for which we may also require $A^{*} \Omega=0, \Omega$ being the vacuum vector.

We call $f(\underline{x}, t): \mathbb{Z}^{d} \times \mathbb{R} \rightarrow \mathbb{C}$ a wave function if for its Fourier transform

$$
\tilde{f}\left(\underline{p}, p_{0}\right):(-\pi, \pi]^{d} \times \mathbb{R} \rightarrow \mathbb{C}
$$

one has

$$
\operatorname{supp} \tilde{f} \cap \operatorname{sp}(H, \underline{P})=\operatorname{supp} \tilde{f} \cap W \neq \emptyset,
$$

where $W=\left\{(\underline{p}, \omega(\underline{p})), \underline{p} \in(-\pi, \pi]^{d}\right\}$ is the "one-particle shell."

Like Hepp [2] consider for a wave function $f$ with $\tilde{f} \in \mathscr{D}\left((-\pi, \pi]^{d} \times \mathbb{R}\right)$ the wave packet $f^{(s)}(x, t)$, parametrized by $s \in \mathbb{R}$, defined as the Fourier transform of $e^{i\left(p_{0}-\omega(p)\right) s} \tilde{f}\left(\underline{p}, p_{0}\right)$, with $\left(\underline{p}, p_{0}\right) \in(-\pi, \pi]^{d} \times \mathbb{R}$.

Set, for $B \in \mathscr{A}$,

$$
B_{f}=\sum_{x \in \mathbb{Z}^{d}} \int_{R} d t f(\underline{x}, t) B(t, \underline{x}),
$$

and

$$
B_{f}(s)=\sum_{x \in \mathbb{Z}^{d}} \int_{R} d t f^{(s)}(\underline{x}, t) B(t, \underline{x}),
$$

for the space-time translated operators $B(t, \underline{x})=e^{i H t} U(\underline{x}) B e^{-i H t} U(-\underline{x})$. If $A \in \mathscr{A}$ is such that $A_{f} \Omega \in \mathscr{D}^{(1)}$, then $A_{f}(s) \Omega=A_{f} \Omega$.

Let $\Delta$ be a closed set in $(-\pi, \pi]^{d}$. Then the velocity content $V(\Delta)$ of the set of momenta $\Delta$ is

$$
V(\Delta)=\{\operatorname{grad} \omega(\underline{p}) \mid \underline{p} \in \Delta\} .
$$

We define also for a wave function $f$,

$$
\Delta(f)=\left\{\underline{p} \in(-\pi, \pi]^{d} \mid(\underline{p}, \omega(\underline{p})) \in \operatorname{supp} \tilde{f} \cap W\right\} .
$$

The following proposition summarises the relevant behavior of the functions $f^{(s)}(\underline{x}, t)$. For a proof see [6]. 
Proposition 1. For $f^{(s)}$ as above with $\tilde{f} \in \mathscr{D}\left(\mathbb{R}^{d+1}\right)$ we have:

a) For all $N \in \mathbb{N}$ there are constants $C_{N}>0$ so that

$$
\left|f^{(s)}(\underline{x}, t)\right| \leqq C_{N}(1+|s|)^{-d / 2}(1+|t-s|)^{-N},
$$

uniformly in $\underline{x}$.

b) There exists a positive constant $C$ so that for every $s$,

$$
\sum_{x \in \mathbb{Z}^{d}} \int_{-\infty}^{\infty} d t\left|f^{(s)}(\underline{x}, t)\right| \leqq C(1+|s|)^{d / 2}
$$

c) For all $L, N \in \mathbb{N}$ there is a positive constant $C_{L, N}$ so that,

$$
\left|f^{(s)}(\underline{x}, t)\right| \leqq C_{L, N}(1+|s-t|)^{-L}(1+\operatorname{dist}(\underline{x}, s V(\Delta(f))))^{-N} .
$$

Now let $\phi_{1}, \ldots, \phi_{n} \in \mathscr{D}^{(1)}$ such that $V\left(\operatorname{sp}_{U} \phi_{i}\right) \cap V\left(\operatorname{sp}_{U} \phi_{j}\right)=\emptyset$ for $i \neq j, \operatorname{sp}_{U} \phi$ is the momentum spectrum of $\phi \in \mathscr{D}^{(1)}$. Choose $A \in \mathscr{A}$ with $A_{f_{i}} \Omega=\phi_{i}, A_{f_{i}}^{*} \Omega=0$ and $\widetilde{f}_{i} \in \mathscr{D}\left((-\pi, \pi]^{d} \times \mathbb{R}\right)$ with non-overlapping velocities:

$$
V\left(\Delta\left(f_{i}\right)\right) \cap V\left(\Delta\left(f_{j}\right)\right)=\emptyset
$$

for $i \neq j$.

To simplify we will often denote $A_{f_{i}}$ by $A_{i}$.

The Haag-Ruelle approximant on the scattering state of particles with single particle states $\phi_{1}, \ldots, \phi_{n}$ is defined by

$$
\phi_{1, \ldots, n}(s)=A_{1}(s) \ldots A_{n}(s) \Omega .
$$

The following theorem on the existence of asymptotic multi-particle states [here denoted by $\left(\phi_{1} \times \ldots \times \phi_{n}\right)_{\text {in }}$ in $]$ and their statistics has been proven in $[1]^{1}$, according to the methods of the Haag-Ruelle Scattering Theory:

Theorem 1. (i) The Haag-Ruelle approximants $\phi_{1, \ldots, n}(s)$ in (9) converge for $t \rightarrow \pm \infty$.

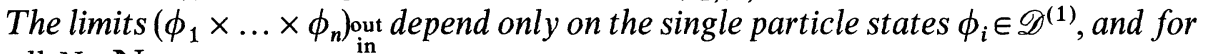
all $N \in \mathbb{N}$,

$$
\left.\| \phi_{1, \ldots, n}(s)-\left(\phi_{1} \times \ldots \times \phi_{n}\right)_{\text {in }}\right)^{\text {out }} \| s^{N} \rightarrow 0, \quad s \rightarrow \pm \infty .
$$

(ii) Let $\psi_{1}, \ldots, \psi_{k} \in \mathscr{D}^{(1)}$ with $V\left(\operatorname{sp}_{U} \psi_{i}\right) \cap V\left(\operatorname{sp}_{U} \psi_{j}\right)=\emptyset, i \neq j$. Then

$$
\left(\left(\psi_{1} \times \ldots \times \psi_{k}\right)_{\text {in }}^{\text {out }},\left(\phi_{1} \times \ldots \times \phi_{n}\right)_{\text {in }}^{\text {out }}\right)=\delta_{n, k} \sum_{\sigma} \prod_{i}\left(\psi_{i}, \phi_{\sigma(i)}\right),
$$

where the sum is over elements $\sigma$ of the permutation group of $\{1, \ldots, n\}$.

This is a consequence of the sufficiently strong clustering property for the Wightman functions that has been established in [1] for the systems in question in the following form:

Theorem 2. Let $B_{1}, \ldots, B_{n} \in \mathscr{A}, n \geqq 2$. Then for the Wightman functions

$$
\mathscr{W}^{(n)}\left(\underline{x}, t_{1} ; \ldots ; \underline{x}_{n}, t_{n}\right):=\left(\Omega, B_{1}\left(t_{1}, \underline{x}\right) \ldots B_{n}\left(t_{n}, \underline{x}_{n}\right) \Omega\right)
$$

1 The fields $A_{f}(s)$ used in the Haag-Ruelle approximants in [1] actually differ slightly from (2). This does not change the result 
the following clustering property holds: for each $\varepsilon, 0<\varepsilon<1$ and for each $q \in \mathbb{N}$, there is a positive constant $C_{q, \varepsilon}$, depending on the $B_{i}$ 's, so that

$$
\left|\mathscr{W}_{T}^{(n)}\left(\underline{x}_{1}, t_{1} ; \ldots ; \underline{x}_{n}, t_{n}\right)\right| \leqq C_{q . \varepsilon} \frac{(1+\|t\|)^{q}}{(1+\|\underline{x}\|)^{(q-1)-\varepsilon}},
$$

where $\|t\|:=\max _{1 \leqq i \leqq n-1}\left|t_{i+1}-t_{i}\right|$ and $\|\underline{x}\|:=\max _{i, j \in\{1, \ldots, n\}}\left|\underline{x}_{i}-\underline{x}_{j}\right| \cdot \mathscr{W}_{T}^{(n)}$ are the truncated n-point Wightman functions.

We will also make use of the following result:

Proposition 2. Let $s \in \mathbb{R}, s<-1$. Hence for all $N \in \mathbb{N}$ there exists a positive constant $C_{M}$ so that

$$
\left\|A_{1}(s)\left(\phi_{2} \times \ldots \times \phi_{n}\right)_{\text {in }}-\left(\phi_{1} \times \ldots \times \phi_{n}\right)_{\text {in }}\right\| \leqq C_{M}|s|^{-M} .
$$

An analogous result holds for out states for $s>1$.

Proof. According with (6) $\|A(s)\| \leqq C(1+|s|)^{d / 2}$. We have

$$
\begin{aligned}
& A_{1}(s)\left(\phi_{2} \times \ldots \times \phi_{n}\right)_{\text {in }}-\left(\phi_{1} \times \ldots \times \phi_{n}\right)_{\text {in }} \\
& \quad=A_{1}(s)\left(\left(\phi_{2} \times \ldots \times \phi_{n}\right)_{\text {in }}-\phi_{2, \ldots, n}(s)\right)-\left(\left(\phi_{1} \times \ldots \times \phi_{n}\right)_{\text {in }}-\phi_{1, \ldots, n}(s)\right),
\end{aligned}
$$

where $\phi_{2, \ldots, n}(s)=A_{2}(s) \ldots A_{n}(s) \Omega$. Hence,

$$
\begin{aligned}
& \left\|A_{1}(s)\left(\phi_{2} \times \ldots \times \phi_{n}\right)_{\text {in }}-\left(\phi_{1} \times \ldots \times \phi_{n}\right)_{\text {in }}\right\| \\
& \leq\left\|A_{1}(s)\right\|\left\|\left(\phi_{2} \times \ldots \times \phi_{n}\right)_{\text {in }}-\phi_{2, \ldots, n}(s)\right\| \\
& \quad+\left\|\left(\phi_{1} \times \ldots \times \phi_{n}\right)_{\text {in }}-\phi_{1, \ldots, n}(s)\right\| \leqq C_{M}|s|^{-M}
\end{aligned}
$$

for $s<-1$ for all $M \in \mathbb{N}$, according with Theorem 1 .

We will use the following definition for the time-ordering of products of fields. ${ }^{2}$

Definition. Let $\sigma_{a_{1}, \ldots, a_{b}}$ be the permutation group of $\left\{a_{1}, \ldots, a_{b}\right\} \subset\{1, \ldots, n\}, n \geqq 1$. Define the time-ordering operation on products of fields $A$ by

$$
T_{\beta}\left[\prod_{i=1}^{b} A\left(t_{a_{i}}, \underline{x}_{a_{\imath}}\right)\right]:=\sum_{\pi \in \sigma_{a_{1}, \ldots, a_{b}}} \prod_{i=1}^{b-1} \theta_{\beta}\left(t_{\pi\left(a_{i}\right)}-t_{\pi\left(a_{i+1}\right)}\right) \prod_{j=1}^{b} A\left(t_{\pi\left(a_{j}\right)}, \underline{x}_{\pi\left(a_{j}\right)}\right),
$$

with $\theta_{\beta}=\theta * \beta$, where $\beta \in \mathscr{D}(\mathbb{R}),{ }^{3}$ with $\int_{-\infty}^{\infty} \beta\left(s^{\prime}\right) d s^{\prime}=1$ and $\beta(x)=\beta(-x)$ and where

$$
\theta(x)= \begin{cases}1, & x>0 \\ 0, & x \leqq 0\end{cases}
$$

Another definition that will be used is:

Definition 2. For $a, b \in \mathbb{N}, b \geqq a$, define

$$
\Gamma_{a, b}=\sum_{\underline{x}_{a}, \ldots, \underline{x}_{b} \in \mathbb{Z}^{d}} \int_{\mathbb{R}^{b}-a+1}\left\{d t_{i}\right\}_{i=a}^{b} \prod_{j=a}^{b} \dot{f}_{j}^{\left(s_{j}\right)}\left(\underline{x}_{j}, t_{j}\right) T_{\beta}\left[\prod_{k=a}^{b} A\left(t_{k}, \underline{x}_{k}\right)\right],
$$

with $\left\{d t_{i}\right\}_{i=a}^{b}=d t_{a} \ldots d t_{b}$. Above $\dot{f}^{(s)}=\frac{d}{d s} f^{(s)}$. Note that $\Gamma_{a, b}$ depends on $s_{a}, \ldots, s_{b}$.

2 The same results can be obtained with more general time-ordering prescriptions [11]

3 The case $\beta \in \mathscr{S}(\mathbb{R})$ can also be treated but involves irrelevant technical complications 


\section{The Main Theorem}

Following Hepp [2] (see also [7] for a brief account) we will establish the following smoothness theorem, which is the central tool for the derivation of the LSZ reduction formulae performed in Sect. 4. Below $\left\{\mathcal{f}_{i}\right\}_{i=1}^{n}$, for $n>1$, will be a set of wave functions in $\mathscr{D}\left((-\pi, \pi]^{d} \times \mathbb{R}\right)$ satisfying $(8)$ (non-overlapping velocities) with $A_{f_{i}} \Omega=\left(\phi_{i}\right) \in \mathscr{H}^{(1)}$.

Theorem 3. For $\left\{\widetilde{f}_{i}\right\}_{i=1}^{n}$ as above and $0 \leqq k<m \leqq n$,

$$
\left(\left(\phi_{1} \times \ldots \times \phi_{k}\right)_{\text {out }}, \Gamma_{k+1, m}\left(\phi_{m+1} \times \ldots \times \phi_{n}\right)_{\text {in }}\right)
$$

belongs to $\mathscr{S}\left(\mathbb{R}^{m-k}\right)$ as function of $s_{k+1}, \ldots, s_{m}$.

Remark. Above, for $k=0,\left(\phi_{1} \times \ldots \times \phi_{k}\right)_{\text {out }}$ has to be replaced by $\Omega$, and the same for $\left(\phi_{m+1} \times \ldots \times \phi_{n}\right)_{\text {in }}$ if $m=n$.

The next two subsections present the proof of this theorem, which will be finished with the proof of Propositions 5 and 6 below. The LSZ reduction formulae follow directly from Theorem 3 in Sect. 4.

\subsection{Proof of Theorem 3. The First Step}

First we note that we just have to prove the fast decay of (20) since the same argumentation given below holds for the derivatives, because $\frac{d^{n}}{d s^{n}} f^{(s)}$ satisfy Proposition 1 for all $n$.

Due to symmetry, we may restrict to the sector

$$
s_{m} \leqq s_{m-1} \leqq \ldots \leqq s_{k+2} \leqq s_{k+1} .
$$

We also restrict to the case in which $s:=-s_{m} \geqq\left|s_{k+1}\right|$. The argumentation in the case $s_{k+1} \geqq\left|s_{m}\right|$ is analogous.

For some fixed $\delta, \varepsilon>0, \gamma: 0 \leqq \gamma \leqq 1$, two successive variables $s_{i}, s_{i-1}, m \geqq i \geqq k+2$ have to satisfy one of the two conditions below:

1. $s_{i-1}-s_{i} \leqq \varepsilon s^{\gamma}+\delta$,

2. $s_{i-1}-s_{i}>\varepsilon s^{\gamma}+\delta$.

In his analysis [2] Hepp takes $\gamma=1$. In our, case, due to the non-uniform clustering of the Wightman functions for space-like separated points we shall need $\gamma<1$, as will be clear at the end of the proof of Theorem 3. This is the reason to consider this more general situation from the beginning.

We write $s_{i-1} \neq s_{i}$ if 1 is valid and $s_{i-1} \sim s_{i}$ if 2 is valid. In the first case we say that $s_{i-1}$ and $s_{i}$ are "connected" and in the second case "disconnected."

There are two possible situations we have to consider:

A) For some $p, 1 \leqq p \leqq m-k-1, s_{m} \sim s_{m-1} \sim \ldots \sim s_{m-p}$, i.e., $s_{j-1}-s_{j}>\varepsilon s^{\gamma}+\delta$ for $m-p+1 \leqq j \leqq m$.

B) For some $q, 1 \leqq q \leqq m-k-1,\left\{s_{m}, \ldots, s_{m-q}\right\}$ is an isolated cluster of connected variables, i.e., $s_{i-1}-s_{i} \leqq \varepsilon s^{\gamma}+\delta$ for $m \geqq i \geqq m-q+1$ and $s_{m-q-1} \sim s_{m-q}$, i.e., $s_{m-q-1}-s_{m-q}>\varepsilon S^{\gamma}+\delta$.

The next two propositions show two convenient approximations for (20) under the cases $\mathrm{A}$ and $\mathrm{B}$ above. 
Proposition 3. Under condition A, expression (20) may be approximated by

$$
\left(\left(\phi_{1} \times \ldots \times \phi_{k}\right)_{\text {out }},\left(\Gamma_{k+1, m-1}\right) \frac{d}{d s_{m}} A_{f_{m}}\left(s_{m}\right)\left(\phi_{m+1} \times \ldots \times \phi_{n}\right)_{\text {in }}\right),
$$

with an error which falls faster than any power in $s$.

Proposition 4. Under condition $\mathrm{B}$, holding for some $q, 1 \leqq q \leqq m-k-1,(20)$ may be approximated by

$$
\left(\left(\phi_{1} \times \ldots \times \phi_{k}\right)_{\text {out }}, \Gamma_{k+1, m-q-1} \Gamma_{m-q, m}\left(\phi_{m+1} \times \ldots \times \phi_{n}\right)_{\text {in }}\right),
$$

with an error which falls faster than any power in $S$.

Remark. Using Proposition 3 we can show that Theorem 3 follows for case A since by Proposition $2, \frac{d}{d s_{m}} A_{f_{m}}\left(s_{m}\right)\left(\phi_{m+1} \times \ldots \times \phi_{n}\right)_{\text {in }}$ approximates

$$
\left(\left(\frac{d}{d s_{m}} \phi_{m}\right) \times \phi_{m+1} \times \ldots \times \phi_{n}\right)_{\text {in }}=0
$$

faster than any power in $\left|s_{n}\right|=s$. The case B is more involving and will be treated in Subsect. 3.2.

3.1.1. Proof of Proposition 3. First we restrict the integration $\int_{-\infty}^{\infty}(\cdot) d t_{m}$ in $(20)$ to an integration on the interval $[-\infty, b(s)]$ with $b(s):=s_{m}+\varepsilon^{\prime} s^{\gamma^{\prime}}+\delta^{\prime}$, for $\varepsilon^{\prime}, \delta^{\prime}>0$, $0 \leqq \gamma^{\prime} \leqq 1$. This introduces an error which is majorized using Proposition 1 by

$$
C_{M}\left[\prod_{i=k+1}^{m}\left(1+\left|s_{i}\right|\right)^{d / 2}\right] \int_{\varepsilon^{\prime} s^{\prime}+\delta^{\prime}}^{\infty} \frac{d y}{(1+|y|)^{M}} \leqq C_{P}^{\prime}|s|^{-P}\left[\prod_{i=k+1}^{m}\left(1+\left|s_{i}\right|\right)^{d / 2}\right],
$$

for any $M \in \mathbb{N}$ for some $P \in \mathbb{N}$ depending on $M$, for $s \geqq 1$. Since $\left|s_{m}\right|=s \geqq\left|s_{i}\right|, \forall i$, the last expression has $C_{Q}^{\prime \prime}|s|^{-Q}$ as upper bound for any $Q \in \mathbb{N}, s>1$, by choosing $P$ large enough.

Now, for all $i, i \neq m$ we restrict the integrations $\int_{-\infty}^{\infty}(\cdot) d t_{i}$ to the interval $[c(s), \infty]$, where $c(s):=s_{m}+\varepsilon^{\prime \prime} s^{\gamma^{\prime}}+\delta^{\prime \prime}$ for $\varepsilon^{\prime \prime}, \delta^{\prime \prime}>0,0 \leqq \gamma^{\prime} \leqq 1$. This introduces an error which is bounded by

$$
C_{M}\left[\int_{-\infty}^{c(s)} \frac{d t_{i}}{\left(1+\left|t_{i}-s_{i}\right|\right)^{M}}\right] \prod_{j=k+1}^{m}\left(1+\left|s_{j}\right|\right)^{d / 2}, \quad \forall M \in \mathbb{N} .
$$

Above the integral can be written as $\int_{-\infty}^{c(s)-s_{\imath}} d y(1+|y|)^{-M}$. Now, since $s_{i} \geqq s_{m-1}(i \neq m)$ we have

$$
c(s)-s_{i} \leqq s_{m}-s_{m-1}+\varepsilon^{\prime \prime} s^{\gamma^{\prime}}+\delta^{\prime \prime}<-\varepsilon S^{\gamma}-\delta+\varepsilon^{\prime \prime} s^{\gamma^{\prime}}+\delta^{\prime \prime},
$$

where in the last inequality we made use of condition A. We see that, choosing $\gamma^{\prime}<\gamma$ or $\gamma^{\prime}=\gamma$ but $\varepsilon>\varepsilon^{\prime \prime}(25)$ becomes bounded by

$$
C_{M}^{\prime} S^{-M}\left(\prod_{j=k+1}^{m}\left(1+\left|s_{j}\right|\right)^{d / 2}\right) \leqq C_{Q}^{\prime \prime} s^{-Q}
$$

for any $Q \in \mathbb{N}$ for some positive constant $C_{Q}^{\prime \prime}$, by choosing $M$ large enough. 
Now choose $\varepsilon^{\prime \prime} \geqq \varepsilon^{\prime}$ and $\delta^{\prime \prime}-\delta^{\prime}>d_{\beta}$, where $d_{\beta}$ is the diameter of $\operatorname{supp} \beta$. With this choice, under the restrictions on the integrations given above, we have for the integration variables $t_{i}-t_{m} \geqq c(s)-b(s)>d_{\beta}, i \neq m$. Due to this inequality we may split $A\left(t_{m}, \underline{x}_{m}\right)$ to the right side in the time-ordered product of $(20)$ and replace it by

$$
\begin{gathered}
\sum_{\left\{x_{a}\right\}_{m=k+1}} \int_{c(s)}^{\infty} \ldots \int_{c(s)}^{\infty} d t_{k+1} \ldots d t_{m-1} \int_{-\infty}^{b(s)} d t_{m} \prod_{i=k+1}^{m} \dot{f}_{i}^{\left(s_{i}\right)}\left(\underline{x}_{i}, t\right) \\
\times\left(\left(\phi_{1} \times \ldots \times \phi_{k}\right)_{\text {out }}, T_{\beta}\left[\prod_{j=k+1}^{m-1} A\left(t_{j}, \underline{x}_{j}\right)\right] A\left(t_{m}, \underline{x}_{m}\right)\left(\phi_{m+1} \times \ldots \times \phi_{n}\right)_{\text {in }}\right)
\end{gathered}
$$

with an error which falls faster than any power in $s$. Returning to full integrations, what costs again an error falling faster than any power in $s$, we complete the proof of Proposition 3.

3.1.2. Proof of Proposition 4. The proof is analogous to the proof of Proposition 3. We first consider the integrations $\int_{-\infty}^{\infty}(\cdot) d t_{i}, m-q \leqq i \leqq m$. We restrict them to the interval $[-\infty, d(s)]$, where $d(s):=s_{m-q}+\varepsilon^{\prime} s^{\gamma^{\prime}}+\delta^{\prime}$. As before, the error is majorized by $C_{M} \int_{d(s)-s_{i}}^{\infty} d y(1+|y|)^{-M}$, for all $M \in \mathbb{N}$, for constants $C_{M}$. Now, since $s_{i} \leqq s_{m-q}($ for $m-q \leqq i \leqq m)$ we have $d(s)-s_{i}=s_{m-q}-s_{i}+\varepsilon^{\prime} s^{\gamma^{\prime}}+\delta^{\prime} \geqq \varepsilon^{\prime} s^{\gamma^{\prime}}+\delta^{\prime}$ and so the last integral has

$$
\int_{\varepsilon^{\prime} s^{\prime}+\delta^{\prime}}^{\infty} \frac{d y}{(1+|y|)^{M}} \leqq C_{N} s^{-N}, \quad N \in \mathbb{N},
$$

as upper bound for a constant $C_{N}>0$, where $N$ can be made arbitrarily large by making $M$ large.

On the other hand, we restrict the integrations $\int_{-\infty}^{\infty}(\cdot) d t_{j}, k+1 \leqq j \leqq m-q-1$ to the interval $[e(s), \infty]$, where $e(s):=s_{m-q}+\varepsilon^{\prime} s^{\gamma^{\prime}}+\delta^{\prime \prime}$ with an error which is bounded by

$$
\int_{-\infty}^{e(s)} \frac{d t_{j}}{\left(1+\mid t_{j}-s_{j}\right)^{M}}=\int_{-\infty}^{e(s)-s_{j}} \frac{d y}{(1+|y|)^{M}} .
$$

Now, note that, since $s_{j} \geqq s_{m-q-1}$ for $k+1 \leqq j \leqq m-q-1$, we have

$$
\begin{aligned}
e(s)-s_{j}= & s_{m-q}-s_{j}+\varepsilon^{\prime} s^{\gamma^{\prime}}+\delta^{\prime \prime} \\
& \leqq s_{m-q}-s_{m-q-1}+\varepsilon^{\prime} s^{\gamma^{\prime}}+\delta^{\prime \prime}<-\varepsilon s^{\gamma}+\varepsilon^{\prime} s^{\gamma^{\prime}}+\delta^{\prime \prime}-\delta
\end{aligned}
$$

(the last inequality coming from condition B) and taking $\gamma^{\prime}<\gamma$ or $\gamma^{\prime}=\gamma$ but $\varepsilon>\varepsilon^{\prime}$ we get for (30) a bound like (29) for $s$ large enough.

Under the restrictions on the integrations given above, for $k+1 \leqq j \leqq m-q-1$ and $m-q \leqq i \leqq m$ we have

$$
t_{j}-t_{i}>e(s)-d(s) \geqq 2 b_{\beta}
$$

by choosing $\varepsilon^{\prime}>\varepsilon$ and, as before, $\delta^{\prime \prime}-\delta^{\prime}>d_{\beta}$. So we get for this case

$$
T_{\beta}\left[\prod_{a=k+1}^{m} A\left(t_{a}, \underline{x}_{a}\right)\right]=T_{\beta}\left[\prod_{a=k+1}^{m-q-1} A\left(t_{a}, \underline{x}_{a}\right)\right] T_{\beta}\left[\prod_{b=m-q}^{m} A\left(t_{b}, \underline{x}_{b}\right)\right] .
$$


The reduction of $\sigma_{k+1, \ldots, m}$ to $\sigma_{k+1, \ldots, m-q-1} \times \sigma_{m-q, \ldots, m}$ is due to the inequality (32) which implies the vanishing of the theta functions involving permutations mixing the groups of variables $\left\{t_{k+1}, \ldots, t_{m-q-1}\right\}$ and $\left\{t_{m-q}, \ldots, t_{m}\right\}$.

Returning to full integrations, what costs an error which again falls faster than any power in $s$, we conclude the proof of the Proposition 4.

\subsection{Proof of Theorem 3. The Last Step}

Now we complete the proof of Theorem 3 by treating (20) for case B using Proposition 4. Our analysis will differ from that of [2] since Hepp makes there use of locality (Einstein locality) to get rid of the time-ordering operation on the fields associated with the connected $s$ variables. Since this property is not available in the systems considered here we have to make use of the clustering of the Wightman functions in a suitable way.

First, one has

$$
\begin{aligned}
& \left|\left(\left(\phi_{1} \times \ldots \times \phi_{k}\right)_{\text {out }}, \Gamma_{k+1, m-1} \Gamma_{m-q, m}\left(\phi_{m+1} \times \ldots \times \phi_{n}\right)_{\mathrm{in}}\right)\right| \\
& \quad \leqq C\left\|\Gamma_{m-q, m}\left(\phi_{m+1} \times \ldots \times \phi_{n}\right)_{\mathrm{in}}\right\|\left[\prod_{i=k+1}^{m-q-1}\left(1+\left|s_{i}\right|\right)^{d / 2}\right],
\end{aligned}
$$

by the Schwarz inequality and Proposition 1. Next we need an upper bound for $\left\|\Gamma_{m-q, m}\left(\phi_{m+1} \times \ldots \times \phi_{n}\right)_{\text {in }}\right\|$. Then we replace $\left(\phi_{m+1} \times \ldots \times \phi_{n}\right)_{\text {in }}$ by its Haag-Ruelle approximant at time $s_{m}$ and define

$$
\Lambda=\Gamma_{m-q, m} A_{m+1}\left(s_{m}\right) \ldots A_{n}\left(s_{m}\right) .
$$

By Theorem 1, $\left\|\Gamma_{m-q, m}\left(\phi_{m+1} \times \ldots \times \phi_{n}\right)_{\text {in }}\right\| \leqq\|\Lambda \Omega\|+R(s)$, where $R(s)$ falls faster than any power in $s$. We concentrate our attention on $\|\Lambda \Omega\|$ whose fast decay will follow directly from the clustering property. After writing $\|\Lambda \Omega\|^{2}$ explicitly and making convenient changes of variables (see below) it can be written as

$$
\begin{aligned}
& \sum_{\left\{z_{a}\right\}_{a}^{2 r}=1} \int_{R^{2 r}}\left\{d \mu_{b}\right\}_{b=1}^{2 r} \prod_{a=1}^{2 r} g_{a}^{\left(v_{a}\right)}\left(\underline{z}_{a}, \mu_{a}\right) \sum_{\substack{\pi^{\prime} \in \sigma_{n-m+1, \ldots, r} \\
\pi \in \sigma_{r+1, \ldots, r+q+1}}} \prod_{k=n-m+1}^{r-1} \theta_{\beta}\left(\mu_{\pi^{\prime}(\varrho(k))}-\mu_{\pi^{\prime}(\varrho(k+1))}\right) \\
& \quad \times \prod_{l=r+1}^{r+q} \theta_{\beta}\left(\mu_{\pi(l)}-\mu_{\pi(l+1)}\right) \mathscr{W}^{(2 r)}\left(\underline{z}_{\hat{\pi}(1)}, \mu_{\hat{\pi}(1)} ; \ldots ; \underline{z}_{\hat{\pi}(2 r)}, \mu_{\hat{\pi}(2 r)}\right)
\end{aligned}
$$

with $r:=n-m+q+1$, where $\varrho \in \sigma_{n-m+1, \ldots, r}$ is the permutation inverting the order of $(n-m+1, \ldots, r): \varrho(i)=r+(n-m+1)-i$. Above the Wightman functions are defined by

$$
\mathscr{W}^{(2 r)}\left(\underline{z}_{1}, \mu_{1} ; \ldots ; \underline{z}_{2 r}, \mu_{2 r}\right):=\left(\Omega, \prod_{a=1}^{r} A\left(\mu_{a}, \underline{z}_{a}\right)^{*} \prod_{b=r+1}^{2 r} A\left(\mu_{b}, \underline{z}_{b}\right) \Omega\right)
$$

and there we used following definitions: for $\pi^{\prime} \in \sigma_{n-m+1, \ldots, r}$ and $\pi \in \sigma_{r+1, \ldots, r+q+1}$,

$$
\hat{\pi}(a)=\left\{\begin{array}{lll}
a, & \text { if } & 1 \leqq a \leqq n-m, \\
\pi^{\prime}(a), & \text { if } & n-m+1 \leqq a \leqq r, \\
\pi(a), & \text { if } & r+1 \leqq a \leqq r+q+1, \\
a, & \text { if } & r+q+2 \leqq a \leqq 2 r,
\end{array}\right.
$$


and

$$
\begin{aligned}
& \left(v_{1}, \ldots, v_{n-m}, v_{n-m+1}, \ldots, v_{r}, v_{r+1}, \ldots, v_{r+q+1}, v_{r+q+2}, \ldots, v_{2 r}\right): \\
& \left(s_{m}, \ldots, s_{m}, s_{m}, \ldots, s_{m-q}, s_{m-q}, \ldots, s_{m}, s_{m}, \ldots, s_{m}\right)
\end{aligned}
$$

and also

$$
\begin{gathered}
\left(g_{1}, \ldots, g_{n-m}, g_{n-m+1}, \ldots, g_{r}, g_{r+1}, \ldots, g_{r+q+1}, g_{r+q+2}, \ldots, g_{2 r}\right): \\
=\left(\bar{f}_{n}, \ldots, \bar{f}_{m+1}, \bar{f}_{m}, \ldots, \bar{f}_{m-q}, \dot{f}_{m-q}, \ldots, \dot{f}_{m}, f_{m+1}, \ldots, f_{n}\right) .
\end{gathered}
$$

Our objective is to show that $\|\Lambda \Omega\|$ falls faster than any power in $s$. To do this we apply the results on the clustering properties of the Wightman functions to expression (36). First, we write the Wightman functions of (36) as a sum over products of truncated vacuum expectation values. In order to consider each relevant case separately one has to split the sum over partitions $\mathscr{P}$ of $\{1, \ldots, 2 r\}$ into two sums:

1. A sum $S_{1}$ over partitions of $\{1, \ldots, 2 r\}$ in sub-sets of two elements, and

2. A sum $S_{2}$ over partitions where at least one sub-set contains more than two elements.

Note that the set $\{1, \ldots, 2 r\}$ contains an even number of elements. The sum $S_{1}$ is given by

$$
\sum_{\mathscr{P}_{2}} \prod_{J \in \mathscr{P}_{2}} \mathscr{W}_{T}^{(2)}\left(\underline{z}_{j_{1}}, \mu_{j_{1}} ; \underline{z}_{j_{2}}, \mu_{j_{2}}\right)
$$

where $\mathscr{P}_{2}=\left\{J_{1}, \ldots, J_{r}\right\}$ is a partition of $\{\hat{\pi}(1), \ldots, \hat{\pi}(2 r)\}$ in sets of two elements in increasing order, i.e., for each $J \in \mathscr{P}_{2}$ we have $J=\left(j_{1}, j_{2}\right)=(\hat{\pi}(a), \hat{\pi}(b))$ with $a<b$. We split the sum $S_{1}$ in two pieces, which we call, respectively, $S_{1}^{\prime}$ and $S_{1}^{\prime \prime}$ :

1 a. A sum $S_{1}^{\prime}$ over all partitions $\mathscr{P}_{2}^{\prime}=\left\{J_{1}, \ldots, J_{r}\right\}$ so that each $J \in \mathscr{P}_{2}^{\prime}$ is of the form $J=\left(j_{1}, j_{2}\right)=(\hat{\pi}(a), \hat{\pi}(b))$ with $1 \leqq a \leqq r$ and $r+1 \leqq b \leqq 2 r$,

and

1b. A sum $S_{1}^{\prime \prime}$ over the rest.

With the next two propositions we complete the proof of Theorem 3.

Proposition 5. The contribution of $S_{1}^{\prime}$ to (36) is zero.

Proposition 6. The contributions of $S_{1}^{\prime \prime}$ and $S_{2}$ to (36) fall faster than any power in $s$.

3.2.1. Proof of Proposition 5. The sum $S_{1}^{\prime}$ is explicitly given by

$$
\sum_{\pi^{\dagger} \in \sigma_{r+1, \ldots, 2 r}} \prod_{a=1}^{r} \mathscr{W}_{T}^{(2)}\left(\underline{z}_{\hat{\pi}(a)}, \mu_{\hat{\pi}(a)} ; \underline{z}_{\pi^{\dagger}(\hat{\pi}(a+r))}, \mu_{\pi^{\dagger}(\hat{\pi}(a+r))}\right) .
$$

After the transformation $\pi^{\dagger} \rightarrow \pi^{\dagger} \circ\left(\hat{\pi}^{-1} \mid(r+1, \ldots, 2 r)\right)$ expression (42) becomes independent of $\pi \in \sigma_{r+1, \ldots, r+q+1}$ [see (38)]. Hence, inserting $S_{1}^{\prime}$ into (36), the expectation values factorize from the sum over $\pi \in \sigma_{r+1, \ldots, r+q+1}$ and one can isolate the factor

$$
\sum_{\pi \in \sigma_{r+1}, \ldots, r+q+1} \prod_{l=r+1}^{r+q} \theta_{\beta}\left(\mu_{\pi(l)}-\mu_{\pi(l+1)}\right)
$$

which turns out to be independent of the variables $\left\{\mu_{j}\right\}_{j=r+1}^{r+q+1}$ due to the symmetry of the function $\beta$. 
To complete the proof of Proposition 5 we perform the sums and integrations over $\left\{\underline{z}_{j}, \mu_{j}\right\}_{j=r+1}^{r+q+1}$, taking into account that (43) is constant. The expression to compute is therefore

$$
\begin{aligned}
& \sum_{\left\{z_{a}\right\}_{a=r+1}^{r+q+1}} \int_{\mathbb{R} q+1}\left\{d \mu_{b}\right\}_{b=r+1}^{r+q+1} \prod_{a=r+1}^{r+q+1} g_{a}^{\left(v_{a}\right)}\left(\underline{z}_{a}, \mu_{a}\right) \\
& \times \sum_{\pi^{\dagger} \in \sigma_{r+1, \ldots, 2 r}} \prod_{a=1}^{r} \mathscr{W}_{T}^{(2)}\left(\underline{z}_{\hat{\pi}(a)}, \mu_{\hat{\pi}(a)} ; \underline{z}_{\pi^{\dagger}(a+r)}, \mu_{\pi^{\dagger}(a+r)}\right),
\end{aligned}
$$

which equals

$$
\sum_{\pi^{\dagger} \in \sigma_{r+1}, \ldots, 2 r} \prod_{a=1}^{r}\left(A\left(\mu_{\hat{\pi}(a)}, \underline{z}_{\hat{\pi}(a)}\right) \Omega,\left(\phi_{\left.g_{\pi^{\dagger}(a+r)}\right)}\right)_{\text {out }}^{\text {in }}\right)=0,
$$

since, after (40), for $r+1 \leqq j \leqq r+q+1$ one has $\left(\phi_{g_{J}}\right)_{\text {out }}^{\text {in }}=0$ because these vectors are of the form $\frac{d}{d s_{i}} \phi_{i}=0, m-q \leqq i \leqq m$. This completes the proof of Proposition 5 .

Remark. For the sums $S_{1}^{\prime \prime}$ and $S_{2}$ there will be for each term at least one factor, a truncated expectation value, smeared with wave functions $\left\{g_{a_{i}}^{\left(v_{a_{1}}\right)}\right\}_{i=1}^{v}, v \geqq 2$, with the property

$$
\bigcap_{i=1}^{v} V\left(\Delta\left(g_{a_{\imath}}\right)\right)=\emptyset
$$

i.e., there are at least two wave functions in $\left\{g_{a_{i}}^{\left(v_{a}\right.}\right\}_{i=1}^{v}$ whose velocities do not overlap. Note that this is not the case for the sum $S_{1}^{\prime}$ since there we may have partitions like $\mathscr{P}_{2}^{\prime}=\{(1,2 r),(2,2 r-1), \ldots,(r, r+1)\}$ coupling pairs of wave functions $\left(\bar{f}_{m}, f_{m}\right), \ldots,\left(\bar{f}_{n}, f_{n}\right)$ to the same 2 -point truncated vacuum expectation value. This is the reason why Proposition 5 is needed.

3.2.2. Proof of Proposition 6. According with the last remark we consider for $v \geqq 2$ expressions of the form

$$
\sum_{\underline{z}_{a_{1}}, \ldots, \underline{z}_{a_{v}}} \int_{\mathbb{R}^{v}} d \mu_{a_{1}} \ldots d \mu_{a_{v}} \prod_{i=1}^{v}\left|g_{a_{i}}^{\left(v_{a_{i}}\right)}\left(\underline{z}_{a_{i}}, \mu_{a_{i}}\right)\right|\left|\mathscr{W}_{T}^{(v)}\left(\underline{z}_{a_{1}}, \mu_{a_{i}} ; \ldots ; \underline{z}_{a_{v}}, \mu_{a_{v}}\right)\right|,
$$

with $\left\{a_{1}, \ldots, a_{v}\right\} \subset\{1, \ldots, 2 r\},\left\{g_{a_{1}}\right\}_{i=1}^{v}$ satisfying (46). First, we restrict the sums $\underline{z}_{a_{i}} \in \mathbb{Z}^{d}$ to sums over $\underline{z}_{a_{i}} \in v_{a_{i}} V\left(\Delta\left(g_{a_{l}}\right)\right)$. This introduces for each $a_{i}$ an error which is majorized, according with Proposition 1, by

$$
C_{N}\left(1+\left|v_{a_{i}}\right|\right)^{-N} \prod_{\substack{j=1 \\ j \neq a_{l}}}^{2 r}\left(1+\left|v_{j}\right|\right)^{d / 2}, \quad \forall N \in \mathbb{N} .
$$

But from condition B and from (39) for any $a_{i}$ we have $v_{a_{i}}-s_{m} \leqq q\left(\varepsilon s^{\gamma}+\delta\right)$ and so for $s$ large enough

$$
\left|v_{a_{i}}\right| \geqq\left|s-q\left(\varepsilon s^{\gamma}+\delta\right)\right|,
$$

implying that (48) falls faster than any power in $s$, by taking $\varepsilon$ small. Using Theorem 2 and Proposition 1, (47) becomes for any $p, N \in \mathbb{N}$,

$$
\begin{aligned}
& C_{p, N}\left[\sum^{\prime}(1+\|\underline{z}\|)^{-(p-1-\varepsilon)}\right] \prod_{i=1}^{v}\left(1+\left|v_{a_{i}}\right|\right)^{-d / 2} \\
& \quad \times\left[\int_{-\infty}^{\infty} \ldots \int_{-\infty}^{\infty} d \mu_{a_{1}} \ldots d \mu_{a_{v}}\left(\prod_{i=1}^{v}\left(1+\left|\mu_{a_{\imath}}-v_{a_{l}}\right|\right)^{-N}\right)(1+\|\mu\|)^{p}\right],
\end{aligned}
$$


where $\|\underline{z}\|=\max \left|\underline{z}_{a_{i}}-\underline{z}_{a_{j}}\right|,\|\mu\|=\max \left|\mu_{a_{i}}-\mu_{a_{i+1}}\right|, C_{p, N}$ is a constant and where $\sum^{\prime}$ is a sum over $\underline{z}_{a_{i}} \in v_{a_{i}} V\left(\Delta\left(\mathrm{g}_{a_{i}}\right)\right)$, $1 \leqq i \leqq v$.

For each $\mu_{a_{i}}$ we proceed the change of variables $\mu_{a_{i}} \rightarrow \mu_{a_{i}}+v_{a_{i}}$ and use the inequality $(1+|x-y|)^{p} \leqq(1+|x|)^{p}(1+|y|)^{p}, \forall p \geqq 0$, to get for the integrals above the bound const $(1+\|v\|)^{p}$. Now, by condition $\mathrm{B}$ and (39)

$$
\|v\|=\max \left|v_{a_{i}}-v_{a_{i+1}}\right| \leqq q\left(\varepsilon s^{\gamma}+\delta\right) \text {. }
$$

To find an upper bound for the sums in (50) we use for $\underline{z}_{a_{i}} \in v_{a_{i}} V\left(\Delta\left(g_{a_{i}}\right)\right), \forall i$, the inequality $\|\underline{z}\| \geqq \min \left|\underline{v}_{a_{i}} v_{a_{i}}-\underline{v}_{a_{j}} v_{a_{j}}\right|$, where the minimum is taken over all $\underline{v}_{a_{i}} \in V\left(\Delta\left(g_{a_{i}}\right)\right), \underline{v}_{a_{j}} \in V\left(\Delta\left(g_{a_{j}}\right)\right)$, for some pair $\left(g_{a_{i}}, g_{a_{j}}\right)$ with $V\left(\Delta\left(g_{a_{i}}\right)\right) \cap V\left(\Delta\left(g_{a_{j}}\right)\right)=\emptyset$. But

$$
\begin{aligned}
\left|\underline{v}_{a_{i}} v_{a_{i}}-\underline{v}_{a_{j}} v_{a_{j}}\right| & =\left|\underline{v}_{a_{i}}\left(v_{a_{i}}-v_{a_{j}}\right)-\left(\underline{v}_{a_{j}}-\underline{v}_{a_{i}}\right) v_{a_{j}}\right| \\
& \geqq|| \underline{v}_{a_{i}}|| v_{a_{i}}-v_{a_{j}}|-| \underline{v}_{a_{j}}-\underline{v}_{a_{i}}|| v_{a j}|| .
\end{aligned}
$$

From condition $\mathrm{B}\left|v_{a_{i}}-v_{a_{j}}\right| \leqq q\left(\varepsilon S^{\gamma}+\delta\right)$ and using (49) we conclude that, choosing $s$ large enough, we will get

$$
\begin{aligned}
& || \underline{v}_{a_{i}}|| v_{a_{i}}-v_{a_{j}}|-| \underline{v}_{a_{j}}-\underline{v}_{a_{i}}|| v_{a_{j}}|| \\
& \quad \geqq\left(s-q\left(\varepsilon s^{\gamma}+\delta\right)\right)\left|\underline{v}_{a_{i}}-\underline{v}_{a_{j}}\right|-\left|\underline{v}_{a_{i}}\right| q\left(\varepsilon s^{\gamma}+\delta\right) \geqq K(1+s)
\end{aligned}
$$

for some constant $K$, by choosing $\varepsilon$ small enough. The sums in (50) are then bounded by

$$
\text { const }\left[\prod_{i=1}^{v}\left(1+\left|v_{a_{i}}\right|\right)^{d / 2}\right](1+s)^{-(p-1-\varepsilon)} .
$$

We conclude from (51) and (54) that (50) has for all $p \in \mathbb{N}$ the upper bound

$$
C_{p}^{\prime} s^{d v / 2}\left(1+s^{\gamma}\right)^{p}(1+s)^{-p+1+\varepsilon}
$$

Taking $\gamma<1$ we see that (50) falls faster than any power in $s$. This completes the proof of Proposition 6 and consequently of Theorem 3.

Remark. Note that (55) is the point where the condition $\gamma<1$ becomes necessary. Its need is a consequence of the fact that in Theorem 2 the clustering property of the Wightman functions was proven for a region smaller than the usual region of space-like separated points.

\section{The Reduction Formulae}

We are able now to derive the LSZ reduction formulae. Using Theorem 3 and the same kind of argument used in the proof of Proposition 3 one shows straightforwardly that

$$
\begin{gathered}
(2 \pi)^{-(d+1) n / 2} \int_{\mathbb{R}^{n}} d s \sum_{\underline{x}_{1}, \ldots, \underline{x}_{n}} \int_{\mathbb{R}^{n}} d t \prod_{i=1}^{n} \dot{f}_{i}^{\left(s_{i}\right)}\left(\underline{x}_{i}, t_{i}\right)\left(\Omega, T_{\beta}\left[\prod_{j=1}^{n} A\left(t_{j}, \underline{x}_{j}\right)\right] \Omega\right) \\
=\sum_{X \subset\{1, \ldots, n\}}(-1)^{|X|}\left(\prod_{a \in\{1, \ldots, n\} \backslash X} A^{\text {out }}\left(f_{a}\right)^{*} \Omega, \prod_{b \in X} A^{\text {in }}\left(f_{b}\right) \Omega\right),
\end{gathered}
$$

where $d s=d s_{1} \ldots d s_{n}, d t=d t_{1} \ldots d t_{n}$ and where $A^{\text {in/out }}(f)$ are creation operators of in/out one-particle states with wave function $f$. 
Since supp $\widetilde{f}_{i}$ contain only the one-particle shell of $\operatorname{sp}(H, \underline{P})$ and since the $f_{i}$ 's are assumed to have non-overlapping velocities, what implies non-overlapping momenta, we conclude the following identity:

$$
\begin{aligned}
& \left(\left(\phi_{1} \times \ldots \times \phi_{m}\right)_{\text {out }},\left(\phi_{m+1} \times \ldots \times \phi_{n}\right)_{\text {in }}\right)=(2 \pi)^{-(d+1) n / 2}(-1)^{n-m} \\
& \quad \times \int_{\mathbb{R}^{n}} d s \sum_{\underline{x}_{1}, \ldots, \underline{x}_{n}} \int_{\mathbb{R}^{n}} d t\left\{\prod_{i=1}^{m} \dot{\bar{f}}_{i}^{\left(s_{l}\right)}\left(\underline{x}_{i}, t_{i}\right)\right\}\left\{\prod_{j=m+1}^{n} \dot{f}_{j}^{\left(s_{j}\right)}\left(\underline{x}_{j}, t_{j}\right)\right\} \\
& \quad \times\left(\Omega, T_{\beta}\left[\prod_{k=1}^{m} A\left(t_{k}, \underline{x}_{k}\right)^{*} \prod_{l=m+1}^{n} A\left(t_{l}, \underline{x}_{l}\right)\right] \Omega\right) .
\end{aligned}
$$

The integrand in $s$ may be written after Fourier transformation as

$$
\begin{aligned}
& \int_{\mathbb{R}^{n(d+1)}} d^{n(d+1)} p\left\{\prod_{i=1}^{m} e^{i\left(\omega\left(\underline{p}_{i}\right)-p_{i}^{0}\right) s_{2}} \overline{\bar{f}}\left(p_{i}\right)\right\}\left\{\prod_{j=m+1}^{n} e^{-i\left(\omega\left(\underline{p}_{j}\right)-p_{j}^{0}\right) s_{j}} \tilde{f}_{j}\left(p_{j}\right)\right\} \\
& \times(2 \pi)^{-(d+1) n / 2}(i)^{n}\left[\prod_{k=1}^{n}\left(\omega\left(\underline{p}_{k}\right)-p_{k}^{0}\right)\right] \tau_{\beta}\left(-p_{1}, \ldots,-p_{m}, p_{m+1}, \ldots, p_{n}\right),
\end{aligned}
$$

where $d^{n(d+1)} p=d^{d+1} p_{1} \ldots d^{d+1} p_{n}$, and where $\tau_{\beta}$ is the distributional Fourier transform of

$$
\left(\Omega, T_{\beta}\left[\prod_{k=1}^{m} A\left(t_{k}, \underline{x}_{k}\right)^{*} \prod_{l=m+1}^{n} A\left(t_{l}, \underline{x}_{l}\right)\right] \Omega\right) .
$$

Note that Theorem 3 tells that

$$
\prod_{k=1}^{n}\left[\omega\left(\underline{p}_{k}\right)-p_{k}^{0}\right] \tau_{\beta}\left(-p_{1}, \ldots,-p_{m}, p_{m+1}, \ldots, p_{n}\right)
$$

is $C^{\infty}$ around the mass shell. Interpreting (58) as a Fourier transformation on the variables $q_{i}^{0}:=p_{i}^{0}-\omega\left(\underline{p}_{i}\right)$ the integrations on $d s$ give

$$
\begin{aligned}
\left(\left(\phi_{1}\right.\right. & \left.\left.\times \ldots \times \phi_{m}\right)_{\mathrm{out}},\left(\phi_{m+1} \times \ldots \times \phi_{n}\right)_{\text {in }}\right) \\
= & i^{n}(2 \pi)^{-(d+1) n / 2} \int_{\mathbb{R}^{n(d+1)}} d^{n(d+1)} p\left\{\prod_{i=1}^{m} \overline{\widetilde{f}}_{i}\left(p_{i}\right)\right\}\left\{\prod_{j=m+1}^{n} \tilde{f}_{j}\left(p_{j}\right)\right\} \\
& \times \prod_{k=1}^{n}\left[\left(\omega\left(\underline{p}_{k}\right)-p_{k}^{0}\right) \delta\left(\omega\left(\underline{p}_{k}\right)-p_{k}^{0}\right)\right] \tau_{\beta}\left(-p_{1}, \ldots,-p_{m}, p_{m+1}, \ldots, p_{n}\right) .
\end{aligned}
$$

The validity of this relation is obvious in the case where the distribution (60) is an element of $\mathscr{S}\left(\mathbb{R}^{n(d+1)}\right)$. The general case follows from this one since $\mathscr{S}$ is weakly dense in $\mathscr{S}^{\prime}$, the dual of $\mathscr{S}$, and since the Fourier transformation is weakly continuous on $\mathscr{S}^{\prime}$ (see also [8, Sect. IX.1]). Expressions (61) are the LSZ reduction formulae in momentum space.

Write $p_{j}^{0} \widetilde{f}_{j}\left(p_{j}\right)=\left(\widetilde{i \partial_{t_{j}}} f_{j}\right)\left(p_{j}\right)$ and $\omega\left(p_{j}\right) \widetilde{f}_{j}\left(p_{j}\right)=\left(\widetilde{E f_{j}}\right)\left(p_{j}\right)$, where '?' denotes Fourier transform and the $E$ is defined by

$$
(E f)(\underline{x}, t):=(2 \pi)^{-n / 2} \int_{(-\pi, \pi]^{d} \times \mathbb{R}} \omega(\underline{p}) \widetilde{f}(p) e^{i\left(\underline{p} \cdot \underline{x}-p_{0} t\right)} d^{d+1} p
$$

and define the distribution $\Delta(\underline{x}, t)$ so that $\widetilde{\Delta}\left(\underline{p}, p_{0}\right)=\delta\left(\omega(\underline{p})-p_{0}\right)$, explicitly:

$$
\Delta(\underline{x}, t)=(2 \pi)^{-(d+1) / 2} \int_{(-\pi, \pi]^{d}} e^{i(\underline{p} \cdot \underline{x}-\omega(\underline{p}) t)} d^{d} \underline{p} .
$$


Then we get

$$
\begin{aligned}
& \left(\left(\phi_{1} \times \ldots \times \phi_{m}\right)_{\mathrm{out}},\left(\phi_{m+1} \times \ldots \times \phi_{n}\right)_{\mathrm{in}}\right)=i^{n}(-1)^{m(d+1)}(2 \pi)^{-(d+1) n / 2} \\
& \quad \times \sum_{\underline{x}_{1}, \ldots, \underline{x}_{n}} \int_{\mathbb{R}^{n}}\left\{d t_{a}\right\}_{a=1}^{n}\left(\Omega, T_{\beta}\left[\prod_{k=1}^{m} A\left(t_{k}, \underline{x}_{k}\right)^{*} \prod_{l=m+1}^{n} A\left(t_{l}, \underline{x}_{l}\right)\right] \Omega\right) \\
& \quad \times\left[\prod_{i=1}^{m} \overline{\left(\Delta *\left(\left(E-i \partial_{t_{i}}\right) f_{i}\right)\right)}\left(\underline{x}_{i}, t_{i}\right)\right]\left[\prod_{j=m+1}^{n}\left(\Delta *\left(\left(E-i \partial_{t_{j}}\right) f_{j}\right)\left(\underline{x}_{j}, t_{j}\right)\right)\right],
\end{aligned}
$$

which are the LSZ reduction formulae in coordinate space.

The main distinction between these formulae and the corresponding ones in relativistic quantum field theories is the use of almost local fields with finite energy transfer in the Wightman functions. This comes from the need to cut out the high energy component of the fields in order to get control on the clustering properties of Wightman functions, as described in [1]. Since in our basic setting no special assumption on the high energy behavior of the theory was made, it is probably not possible in general to eliminate this restriction on the high energy of the fields without violating our basic results. Additional assumptions on the locality properties of the high energy of the fields are then necessary.

An additional remark concerns the time-ordered functions occurring in (61) and (64). In continuum theories, time-ordered functions can be obtained, at least formally, through analytical continuation of Euclidean expectation values (for an analysis in the framework of the Osterwalder-Schrader axioms see [9]). This possibility was studied by Lüscher [4] in the context of Euclidean lattice models of scalar fields, with the result that, for these lattice models, the formally defined timeordered functions and the functions obtained by analytical continuation of Euclidean expectation values differ at asymptotic times by exponentially falling factors. It is presently not clear, whether Lüscher's results may be reproduced for our reduction formulae, since the restriction on the high energy component of the fields is essential in our analysis.

Acknowledgement. I am indebted to Klaus Fredenhagen for the suggestion of this work and for valuable discussions.

\section{References}

1. Barata, J.C.A., Fredenhagen, K.: Particle scattering in Euclidean lattice field theories. Commun. Math. Phys. 138, 507-519 (1991)

2. Hepp, K.: On the connection between the LSZ and the Wightman quantum field theory. Commun. Math. Phys. 1, 95-111 (1965)

Hepp, K.: On the connection between the LSZ and the Wightman quantum field theory. In: Axiomatic field theory. Brandeis 1965. Chretien, M., Deser, S. (eds.) New York: Gordon and Breach 1966

3. Lehmann, H., Symanzik, K., Zimmermann, W.: Zur Formulierung quantisierter Feldtheorien. Il Nuovo Cimento I, 205-225 (1955)

4. Lüscher, M.: Selected topics in lattice field theory. Desy-preprint 88-156. Lectures given at the Summer School "Fields, Strings, and Critical Phenomena", Les Houches, France (1988)

Lüscher, M.: Streutheorie für euklidische skalare Feldtheorien auf einem Raumzeitgitter. Unpublished notes (1985)

5. Lüscher, M., Wollf, U.: How to calculate the elastic scattering matrix in two-dimensional quantum field theory by numerical simulation. Nucl. Phys. B 339, 222 (1990)

Lüscher, M.: Two particle states on a torus and their relation to the scattering matrix. Desypreprint 90-131 (1990) 
6. Reed, M., Simon, B.: Methods of modern mathematical physics. Scattering theory, Vol. III. New York: Academic Press 1972-1979

7. Glimm, J., Jaffe, A.: Quantum physics. A functional integral point of view. Second edition. Berlin, Heidelberg, New York: Springer 1987

8. Reed, M., Simon, B.: Methods of modern mathematical physics. Fourier analysis, selfadjointness, Vol. II. New York: Academic Press 1972-1979

9. Eckmann, J.-P., Epstein, H.: Time-ordered products and Schwinger functions. Commun. Math. Phys. 64, 95-130 (1979)

10. Barata, J.C.A.: Scattering states of charged particles in the $\mathbb{Z}_{2}$ gauge theories. Commun. Math. Phys. 138, 175-191 (1991)

11. Barata, J.C.A.: Scattering theory for Euclidean lattice field theories. Ph.D. Thesis. F.U. Berlin 1989

Communicated by A. Jaffe 